\title{
Reliable Delay Constrained Multihop Broadcasting in VANETs
}

\author{
Martin Koubek, Susan Rea, and Dirk Pesch \\ NIMBUS Centre for Embedded Systems Research, Cork Institute of Technology, Cork, Ireland \\ Correspondence should be addressed to Susan Rea, susan.rea@cit.ie
}

Received 26 November 2009; Accepted 5 September 2010

Academic Editor: Hossein Pishro-Nik

Copyright (C) 2010 Martin Koubek et al. This is an open access article distributed under the Creative Commons Attribution License, which permits unrestricted use, distribution, and reproduction in any medium, provided the original work is properly cited.

\begin{abstract}
Vehicular communication is regarded as a major innovative feature for in-car technology. While improving road safety is unanimously considered the major driving factor for the deployment of Intelligent Vehicle Safety Systems, the challenges relating to reliable multi-hop broadcasting are exigent in vehicular networking. In fact, safety applications must rely on very accurate and upto-date information about the surrounding environment, which in turn requires the use of accurate positioning systems and smart communication protocols for exchanging information. Communications protocols for VANETs must guarantee fast and reliable delivery of information to all vehicles in the neighbourhood, where the wireless communication medium is shared and highly unreliable with limited bandwidth. In this paper, we focus on mechanisms that improve the reliability of broadcasting protocols, where the emphasis is on satisfying the delay requirements for safety applications. We present the Pseudoacknowledgments (PACKs) scheme and compare this with existing methods over varying vehicle densities in an urban scenario using the network simulator OPNET.
\end{abstract}

\section{Introduction}

The US Federal Communications Commission (FCC) and later the European Telecommunications Standards Institute (ETSI) approved a frequency band reservation in the $5.9 \mathrm{GHz}$ (in Europe $5 \mathrm{GHz}$ ) band for wireless communications between vehicles $(\mathrm{V} 2 \mathrm{~V})$ and roadside (V2R) infrastructures. At present, the IEEE group is completing the final drafts of the IEEE 802.11p and IEEE P1609 "Standard for Wireless Access in Vehicular Environments (WAVEs)" [1]. The European Commission through programmes like the i2010 Intelligent Car Initiative [2], which is a followup of eEurope 2005 [3] is driving the rollout of intelligent vehicle systems in both European and international markets, by supporting ICT research and development in the area of transport. Under i2010, eSafety is a collaborative initiative involving the European Commission, industry, and other stakeholders to hasten the development, deployment, and use of Intelligent Vehicle Safety Systems (IVSSs) as a means of increasing road safety and reducing the number of road traffic accidents within Europe.

Integrating a network interface, GPS receiver, sensors, and on-board computers presents an opportunity to build a powerful car-safety system, capable of gathering, processing, and distributing information. By collecting accurate and up-to-date information concerning the status of the surrounding environment, a driver assistance system can quickly detect potentially dangerous situations and notify the driver regarding this impending peril. Notifying other drivers can be achieved via vehicle-2-vehicle (V2V) communications typically relying on broadcasting as the underlying dissemination technique. However, broadcasting is a very expensive dissemination technique that needlessly consumes channel communication capacity with increased collisions and packets losses [4]. A broadcasting protocol for VANETs must guarantee fast and reliable delivery of information to all vehicles in the neighbourhood, where the wireless communications medium is shared, very unreliable, and with limited bandwidth. It must guarantee high delivery rates for priority messages with emergency payload data in all situations from small vehicle densities (rural areas) to crowded roads in cities during peak times with the communication network may be well saturated.

Broadcasting protocols (e.g., [5-9]), that have been proposed for VANETs have a common factor in that they cannot guarantee high reliability for safety-related data 
dissemination with [5] concluding that the probability of successful reception of the data decreases with growing distance from the sender. These factors have serious consequences for safety-related data dissemination where dangerous situations can be aggravated through unsuccessful broadcast communications.

In this paper, we propose a scheme called Pseudoacknowledgments (PACK) that interprets successful multihop broadcast transmission through overhearing successive rebroadcasts by its neighbours. As the broadcast packet traverses the network, each hop creates dynamic time slots in order to rebroadcast. Intermediate hops that receive the broadcast wait until the dynamic slot time expires and then rebroadcasts thereby acknowledging a link between itself and a previous hop. If the previous hop does not overhear the rebroadcast, it repeats the rebroadcasting. The dynamic slots are created locally at individual nodes and do not require a global clock.

The advantage of the PACK method is that it does not need any extra hardware and can be implemented on top of any broadcasting protocol, however, our simulation results have demonstrated that most gains in efficiency are achieved with location-based p-persistent CSMA/CA broadcasting protocols. The PACK schemes rapidly increase reception probability of broadcasting protocols with minimal additional overhead in terms of latency and retransmissions. In this paper, we compare the efficiency of the PACK method with existing schemes for reliable multihop broadcasting that increase the reception probability. The network simulator tool OPNET [10] is used to develop an accurate urban scenario based on the VANET specific WAVE communications protocol with realistic vehicle mobility patterns, radio propagation model using $802.11 \mathrm{p}$.

\section{Related Work}

One of the primary concerns for broadcast protocols lies in the unreliable packet delivery. Protocols such as ALOHA and CSMA are some of the earliest works that focus on mitigating packet collisions in uncoordinated networks. Following on from this CSMA with Collision Avoidance was developed which is the basis for the IEEE 802.11 suite of communications protocols of which IEEE $802.11 \mathrm{p}$ for V2V communications is part of it. An RTS/CTS handshake exchange mechanism has been developed for unicast transmissions to increase reliability, however, broadcast transmissions still have to rely only on pure CSMA/CA protocol without RTS/CTS. A common concern for broadcasting algorithms in VANETs is their inability to achieve a packet reception rate close to $100 \%$ [5].

2.1. Multihop Broadcasting Schemes. For multihop broadcasting protocols, several works have proposed acknowledging techniques to increase reliability in MARQ [11], BACK [12], and BSMA [13] schemes. These methods are based on reserving time slots where a sender allocates virtual time slots for all its neighbours and transmits the broadcast data. All its neighbours transmit ACKs in their virtual slot. The reserving of virtual time slots for individual ACK transmissions is problematic in denser networks as it leads to a dramatic increase in latency, a fundamental concern for the dissemination of safety related data. The authors in [6] proposed a broadcasting protocol called UMB that uses a handshake like RTS, CTS and ACK for onedirectional broadcasting, however, this protocol requires the positioning of intersection repeaters that acknowledge the broadcast along the physical roadways. Other multihop broadcast protocols presented in [7] include V-TRADE and HV-TRADE. A node wishing to transmit or retransmit a broadcast transmits a position request at first to all neighbours and waits until all neighbours reply. After all replies have been received, the node transmits the broadcast with a list of selected nodes that act as forwarders similar to OLSR [14]. This was one of the earlier works to address broadcasting in VANET, and the overhead incurred with the position request and reply packets at each hop can contribute to network congestion in denser networks and also increase delay.

From best of our knowledge, there is no method to increase broadcast reliability in multihop broadcast protocols for VANET networks that do not suffer from dramatically rising latency and/or increased load on the physical medium through numerous redundant transmissions. As a precursor to presenting the proposed PACK method, we discuss the mechanisms previously developed to increase reliability for 1-hop broadcasting.

2.2. 1-Hop Broadcasting Schemes. In [15], the authors have identified protocols that increase the reliability of 1-hop broadcasting schemes and have grouped the schemes based on their channel access methods.

(i) The first group is based on CSMA/CA where protocols (e.g., [11-13]) use a handshake mechanism comprising of short packets similar to RTS/CTS/ACK packets.

(ii) The second group of protocols relies on reserving time slots in the physical medium. For the RRALOHA [16], vehicles must continuously exchange 2-hop information to reserve free time slots without any central coordination units. The RR-ALOHA was proposed within the European research project CarTalk2000 [17].

(iii) The third group relies on the repetition of broadcasting transmissions. The SFR $[18,19]$ protocol randomly repeats broadcasted transmissions. The authors in [15] propose the OOC code that dynamically affects the number of repetition. The OOC method performed better against SFR $[15,20]$, but for fast moving vehicles the OOC protocol has difficulties with codeword synchronisation.

(iv) Another group of protocols not discussed in [15] investigated changing the transmission power of broadcasting messages to control the wireless bandwidth $[21,22]$. The Adaptive Transmission Power 
(ATP) protocol [21] changes the transmit power depending on the number of 1-hop neighbours.

In recent years, several 1-hop broadcasting schemes have been developed for VANETs whereas not many efforts were invested in improving existing multihop broadcasting schemes described in Section 2.1. For safety-related data dissemination, there will be a prerequisite to dissemination data beyond a single hop with high reliability for data delivery over several hops with minimal delay and low data collisions. In this paper, we propose a multihop scheme that

(i) improves the reliability of multihop broadcast protocols,

(ii) with a marginal increase in latency and link load.

The proposed approach is based on creating flexible time slots at the transmitter and the pseudoacknowledging of transmissions by rebroadcasting nodes through overhearing. We choose three 1-hop reception schemes namely RRALOHA, SFR, and ATP that we have extended for use with multihop broadcasting and compared their performance with our proposed scheme we refer to as Pseudoacknowledgments (PACKs). We tested the schemes under different vehicle densities where we emulated local (accidents) and global (raining) events. An urban environment has been selected for experimental evaluation as opposed to a rural or motorway scenario as this environment will be densely populated with slower moving vehicles that force the use of multihop broadcast protocols as transmission distances are severely attenuated with obstacles present in the environment such as buildings, traffic lights, and restricted roadways that cause a build up and congestion in traffic flows.

\section{Multihop Broadcast Protocol}

The methods for increasing multihop broadcast protocol reliability have been overlaid on the same underlying base broadcast protocol namely the low latency Slotted Restricted Mobility-Based (SRMB) protocol as opposed to using flooding. The SRMB protocol is an extension of the Restricted Mobility-Based (RMB) [23] broadcasting protocol with SRMB minimising data collisions on forwarding broadcasts by using a dynamic slot wait time generated in the upper MAC layer in the order of milliseconds. PACK can be used with any broadcasting protocol, but dynamic slot wait times (SRMB) have been shown to reduce collisions by modifying the channel access times. Protocols, which include already some form of slot wait times, for example, in case of AODV [24] and OLSR [14] random wait time in the range of 0 to $100 \mathrm{~ms}$, do not need necessary integrate SRMB extensions to use PACK.

The RMB, SRMB, and PACK algorithms are described next, prior to the presentation of the experimental evaluations.

3.1. Restricted Mobility-Based (RMB) Protocol. We have previously presented the RMB algorithm in [23]. RMB is a flat (nonclustered), uncentralised, p-persistent CSMA/CA

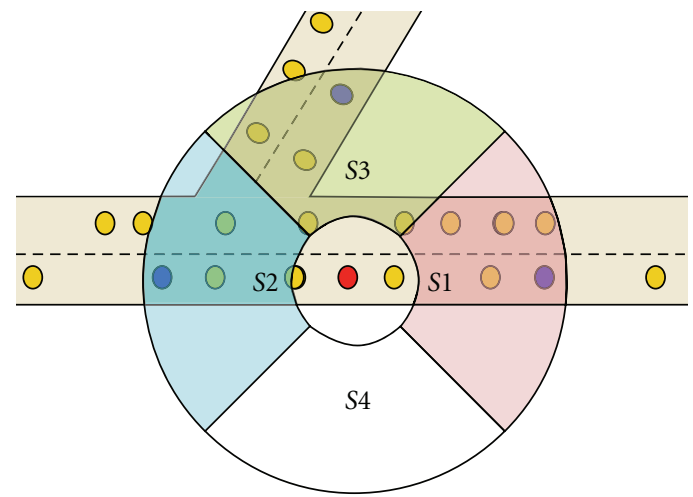

Figure 1: Directional sectors are defined about the transmitting node with a radius defined by the theoretical transmission distance with each sector having a $90^{\circ}$ spread.

broadcasting protocol that reduces redundant broadcast transmissions using 1-hop location knowledge obtained from beacons. RMB was compared with the DV-CAST protocol [25], with RMB having fewer transmissions, lower end-to-end delay, and a high delivery ratio.

The basic principle of this algorithm is that before broadcasting (rebroadcasting) a transmitter $M_{i}$ determines a small set of its neighbours MPR ${ }_{1 \cdots N}^{i}$ (Multipoint Relay set as used in OLSR [14]) with each node lying in a geographically different sector (maximum $N \leq 4$ sectors)

as shown in Figure 1. The transmitter records the shortened MAC addresses of the $\mathrm{MPR}_{1 \cdots N}^{i}$ nodes in the packet header and broadcasts. A node $M_{j}$ that receives the packet and has its MAC address recoded in the packet header assigns a Backoff time slot "0" for rebroadcasting in the MAC buffer. A node $M_{k}$, which receives the packet and finds that its MAC address does not match any address recorded in the packet header, assigns its Backoff time slot depending on its position, speed, and motion vector compared against the transmitter in range from 1 to the maximum value of the particular Contention Window (CW). The maximum size of CW depends on the type of traffic (voice, video, and data) and ranges from 3 to $15[1,26]$. A Backoff time slot of "1" refers to nodes that are sufficiently far from the transmitter and have similar speed and motion vector as the transmitter. Larger Backoff time values indicate that nodes have different motion vectors and speeds compared to the transmitter [23, Section 3].

To avoid redundant transmissions during broadcasting, each node $M_{i}$ (MPR, non-MPR) assesses whether all of its neighbours have received the broadcast packet. This is based on the knowledge of the position of the transmitter and all neighbours and the knowledge of transmission distance. If all neighbours are assessed by the $M_{i}$ to have received the broadcast and the $M_{i}$ has the same broadcast to transmit, then $M_{i}$ discards the packet and does not rebroadcast.

The RMB scheme ensures that during broadcasting if a collision occurs at an MPR node, some other non-MPR node with the second highest priority substitutes as the MPR and rebroadcasts. A strong advantage of this scheme lies in 


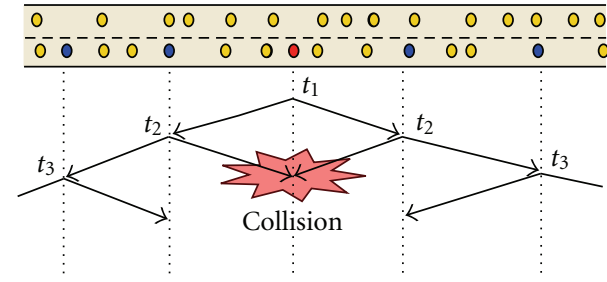

FiguRE 2: RMB.

the fast broadcasting process where nodes wait for retransmitting generally less than a millisecond. A disadvantage is that the contention window size of a traffic class may not be sufficiently large enough to transmit without collisions at non-MPRs, for example, considering the "Voice" traffic class, there are only 3 Backoff time slots. This implies that nonMPR nodes can, with a high probability, be assigned the same time slot which leads to collisions, thus, effectively stopping the broadcast.

3.2. SRMB and PACK. The RMB algorithm suffers from the hidden terminal problem as illustrated in Figure 2.

A source node $M_{i}$ broadcasts at time $t_{1}$ with MPR set $\mathrm{MPR}_{j, k}^{i}$. All neighbours of $M_{i}$ that received the broadcast and because MPR nodes $\left(M_{j}\right.$ and $\left.M_{k}\right)$ set the Backoff time to " 0 " and are not within transmission range of each other (Hidden Terminal problem), $M_{j}$ and $M_{k}$ rebroadcast at time $t_{2}$. Because $M_{j}$ and $M_{k}$ transmit in the same time (or within a short proximity), a collision occurs around $M_{i}$ in $t_{2}$. The collision generally does not have an effect on surrounding nodes of $M_{i}$ because these nodes already received the broadcast in time $t_{1}$. But $M_{i}$ does not overhear (receive) the broadcasts sent by $M_{j}$ and $M_{k}$ correctly and so $M_{i}$ does not know if its own broadcast transmission was successfully received at its neighbours.

To minimise collisions at the source node (and likewise at intermediate nodes that act as forwarders), we developed the Slotted Restricted Mobility-Based (SRMB) algorithm and the Pseudoacknowledgments (PACK) scheme.

3.2.1. Slotted Restricted Mobility-Based (SRMB) Algorithm. The main contribution of the SRMB extension is that rebroadcasting is carefully scheduled (spread in time) using dynamic slot wait times (Figure 3). Each node that receives a broadcast packet assigns a dynamic wait time slot for rebroadcasting to ensure that nodes have sufficient time for rebroadcasting. The wait time slot is derived from the maximum transmission time $T_{L_{-} \text {MAC }}(1)$ including processing at lower MAC layer and the time needed for transmission

$$
\begin{aligned}
T_{L_{-} \text {MAC }}(\mathrm{ac})= & \frac{L_{\text {DATA }}}{R_{\text {DATA }}}+\frac{D}{c}+\text { SIFS } \\
& +\cdot T_{\text {BoSlot }} \cdot(\text { AIFSN }+ \text { CW }[\mathrm{ac}]) .
\end{aligned}
$$

(The equation is valid only for lightly loaded networks, In busier networks, if a transmission is heard while a node is in Backoff, then the new Backoff time is set and transmission delay (1) is increased.)
TABle 1: Parameters in different traffic categories.

\begin{tabular}{lcc}
\hline Access Category & AIFSN & CW $_{\max }$ \\
\hline CW $[$ background $\sim$ WSA $]$ & 7 & 15 \\
CW $[$ voice $\sim$ WSM $]$ & 2 & 3 \\
\hline
\end{tabular}

(i) $L_{\text {DATA }}$ is the size of data transmitted over the physical medium in bits. It contains the data payload, WAVE, and MAC headers.

(ii) $R_{\text {DATA }}$ is the data rate in bits per second.

(iii) $D$ is the theoretical distance within which packets can be successfully received. This depends on the environment radio propagation characteristics. In our simulation, we set the transmission distance to $200 \mathrm{~m}$, which has been determined from empirical data measurements and is described in Section 4.

(iv) $c$ is the speed of light set to $3 \times 10^{8} \mathrm{~m} / \mathrm{s}$.

(v) SIFS is the short interframe space with a length of $16 \mu \mathrm{s}$.

(vi) AIFSN specifies the number of "slot" periods within the AIFS (Arbitration Interframe Space) value used by an access category during contention (Table 1 ).

(vii) AIFS is the lag time between the medium becoming idle and the time when the access category starts or resumes a random Backoff period.

(viii) $\mathrm{CW}$ is a number of slots in particular Contention Window (Table 1).

(ix) ACs are the Access Categories used by 802.11e and WAVE MAC to manage different traffic classes (voice, video, and data).

(x) $T_{\text {BoSlot }}$ is the duration of a slot, this is set to $9 \mu \mathrm{s}$.

The SRMB algorithm extends the RMB principle and works as follows.

A station $M_{j}$ receives the packet and encapsulates the list of $\mathrm{MPR}_{1 \ldots N}^{i}$ addresses from the incoming packet. If any addresses of $\mathrm{MPR}_{1 \cdots N}^{i}$ match the $M_{j}$ address, then before a retransmission $M_{j}$ adds a delay in length of wait time slot $T_{\text {slot }}$ as follows:

$$
T_{\text {slot }}(J)=(J-1) \cdot m \cdot \max \left(T_{L \_ \text {MAC }}\right) .
$$

(i) $J$ is $J \in(1 \leq N)$ and is the order of the node $M_{j}$ in the list of $\mathrm{MPR}_{1 \cdots N}^{i}$.

(ii) $m$ is a multiplier added to avoid collisions when the networks become busy and (1) expires. The value is set to 1.5 , which has been determined from simulation investigation.

Else if $M_{j}$ address does not match any of the addresses in $\mathrm{MPR}_{1 \cdots N}^{i}$, then before a transmission node $M_{j}$ adds a time delay according to (2), where

(i) $J=N+S$; 


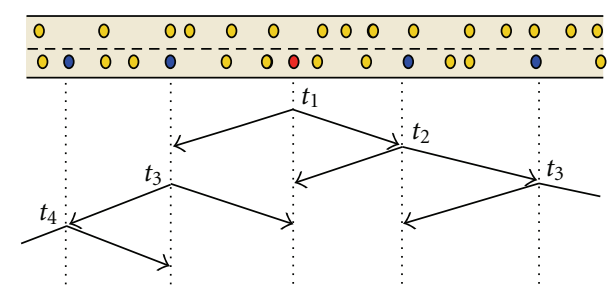

FIGURE 3: SRMB.

(ii) $N$ is the maximum number of nodes in $\mathrm{MPR}_{1 \ldots N}^{i}$;

(iii) $S$ is the order of the sector where $M_{j}$ is positioned (Figure 1). A sector is defined about the transmitting node with a radius defined by the theoretical transmission distance with each sector having a $90^{\circ}$ degree spread [23].

Time slots are chosen based on an MPR node priority, and MPR nodes transmit one by one leaving sufficient time to avoid collisions at a source node and also to avoid collisions between other non-MPR nodes in different sectors.

3.2.2. Pseudoacknowledgments (PACK). The principle of SRMB is that nodes $M_{j \cdots n}$ broadcast one by one without collisions at the source node or previous forwarding hop $M_{i}$. As previously described, a broadcasting node defines geographical sectors and selects its MPR set $\mathrm{MPR}_{1 \ldots N}^{i}$ and broadcasts. Selected neighbours of $M_{i}$ that receive the broadcast say $M_{j}$ and $M_{k}$ then rebroadcast. The rebroadcasting by $M_{j}$ and $M_{k}$ is also received (overheard) at $M_{i}$ (Figure 3) assuming no collisions. Collisions are mitigated due to the spreading of the retransmissions over dynamic wait time slots, and so each rebroadcast node should transmit in turn and be overheard by $M_{i}$. This overhearing is interpreted by the PACK method as a form of pseudoacknowledgement for the individual sectors. If an unacknowledged sector(s) remains after some predefined time (as per (3)), then the node $M_{i}$ repeats the broadcast with a new list of $\mathrm{MPR}_{1 \cdots M}^{i}$ that contains only the missing sector(s). The algorithm is repeated until all sectors are acknowledged or a maximum number of repetitions are reached for the broadcast. The broadcast repetition interval $T_{\text {rep }}$ is calculated according to the following equation:

$$
\begin{aligned}
T_{\text {rep }}= & 2 \cdot N \cdot \max \left(T_{L-M A C}\right) \\
& + \text { Rand }\left(N \cdot \max \left(T_{L-M A C}\right)\right) .
\end{aligned}
$$

(i) $N$ is the maximum number of nodes in $\mathrm{MPR}_{1 \ldots N}^{i}$. For other broadcasting protocols other than SRMB, $N$ represents the number of nodes that can possibly retransmit.

(ii) Rand is a random value uniformly distributed in the range 0 to $\left(N \cdot \max \left(T_{L M A C}\right)\right)$ to further randomise repetitions over a short time interval to avoid collisions.

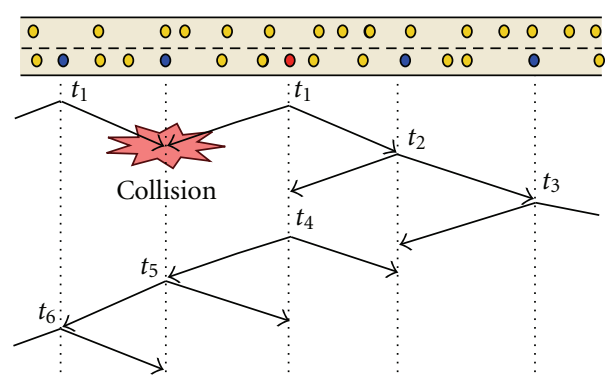

Figure 4: SRMB+PACK.

The PACK scheme partly solves the Hidden Terminal Problem by using repetitions Figure 4. The maximum repetitions are set to 3 by default.

The fundamental difference between SRMB+PACK and slotted protocols such as RR-ALOHA is that SRMB+PACK uses access CSMA to the physical medium. Only specific nodes act as forwarders for the broadcast and in turn create virtual time slots during the broadcasting process at the upper MAC layer to further randomise the channel access time to decrease packet collisions. Nodes set the start of the repetition slots based on the time the packet is received so global synchronisation is not required and the slot size is determined using (3). After this wait time slot expires, the broadcast packet is passed from the upper MAC layer to the lower MAC layer for transmission according to the particular MAC standard (e.g., [26]).

The converse is true for slotted protocols such as RR-ALOHA, where TDMA is used to access the physical medium. All nodes must rely on a global clock for synchronization, and each node has its own reserved time slot to transmit with a fixed length, which makes this scheme unsuitable for variable length packets or event/bursty traffic.

3.3. Reliable Broadcast Schemes under Test. In this paper, we compared the proposed multihop SRMB+PACK scheme with 3 other reliable broadcast methods. These mechanisms also used SRMB as the underlying broadcasting mechanism with WAVE [1] as the communications protocol.

3.3.1. Synchronous Fixed Retransmission (SFR). SFR has been presented in $[18,19]$ and is based on repetitively broadcasting the same message by a sender. The number of rebroadcasts is not constant and is randomly chosen according to the following principle.

Messages are assumed to have a specific lifetime, and this life time, is divided into time slots, and from this a random number of these slots are chosen to repetitively transmit the broadcast. The time slots are synchronized to a global clock. The authors have proposed other mechanisms but have shown that SFR achieves the better performance.

In [18], the message lifetime is set to $100 \mathrm{~ms}$, which we see as unsuitable because it significantly lengthens safety messaging. Considering this, we decreased the lifetime to $10 \mathrm{~ms}$ and derived a suitable slot length of $1 \mathrm{~ms}$ using (1) and (2), giving sufficient time to perform repetitive broadcasting. 
Each sender can randomly choose from 0 to 9 repetitions for broadcasting and then broadcast in the selected slot.

3.3.2. Adaptive Transmission Power for Beacons (ATPBs). The Adaptive Transmission Power (ATP) protocol presented in [21] is based on nodes listening to the medium and counting the collisions that occur in this period. Depending on this value and the number of neighbours, a node decreases or increases its transmission power appropriately. In [21], the threshold for the number of neighbours is set to 30 , when this value is exceeded the transmit power is controlled using ATP.

Irrespective of the packet type, the same power is used to transmit all messages. Authors [27] highlight that such an approach leads to dangerously reduced transmission ranges for emergency data and this is counter productive, where emergency data is typically sent on the maximum transmit power to cover as many nodes as possible. Improved performance is achieved using the maximum transmit power as opposed to broadcasting over multiple hops. As an alternative to ATP, we developed the Adaptive Transmission Power for Beacons (ATPBs), which relies on the same method of assessing channel, but the transmit power is only modified for the periodic beacons to spare communication capacity for safety messages that are transmitted with the maximum possible transmit power.

3.3.3. Reliable Reservation-ALOHA (RR-ALOHA). The RRALOHA protocol presented in [16] has been developed within the European research project CarTalk2000 [17]. This is a slotted technique (TDMA access), where nodes rely on synchronised time slots for communications, where nodes are assigned a single dedicated slot for transmission. To prevent nodes from using the same slot, the ReservationALOHA (R-ALOHA) [28] protocol uses a central repeater that announces used slots. This concept is impracticable for use within VANETs because the inclusion of static infrastructure would restrict VANET communications to centralised vehicle-2-Infrastructure communications. To avoid the use of central repeaters, RR-ALOHA [16] was developed and proposes that each node sends beacons containing information identifying which slot is used for communications with their 1-hop neighbours. A node, which receives beacons from its 1-hop neighbour nodes, indirectly receives information identifying the used slots for its 2-hop neighbours. This allows nodes to access free slots and to avoid the Hidden Terminal Problem.

\section{Simulation Environment}

We have developed a VANET simulation environment using the network simulation tool OPNET V.12 [10] to evaluate the performance of the PACK algorithm over the Slotted Restricted Mobility-Based (SRMB) broadcast algorithm and integrated this with the VANET specific Wave Short Message Protocol (WSMP) based on a simplified model of the Wave communications standard (parameters are shown in Table 2). The Wave model contains one Control Channel
TABLE 2: Scenario description.

\begin{tabular}{ll}
\hline Scenario & Urban scenario \\
\hline Transmit power & $18 \mathrm{dBm}$ \\
Frequency & $5.9 \mathrm{GHz}$ \\
Data rate & $6 \mathrm{Mbit} / \mathrm{s}$ \\
Bandwidth of channel & $10 \mathrm{MHz}$ \\
Transmit range & Two-Ray Ground model \\
Minimum broadcast distance & with shadowing \\
Maximum num hops & $500 \mathrm{~m}$ \\
Speed of vehicles & 10 \\
Scenario dimensions & $0-50 \mathrm{~km} / \mathrm{h}$ \\
Density of nodes/km ${ }^{2}$ & $2 \mathrm{~km} \times 2.5 \mathrm{~km}$ \\
Number of Hazardous locations & $10-140$ \\
Repetition interval of safety messages & 3 (accident), 1 (rain) \\
Size of beacons & $480 \mathrm{~b}$ \\
Size of safety messages & $368 \mathrm{~b}$ \\
Beaconing interval & $100 \mathrm{~ms}$ \\
\hline
\end{tabular}

$(\mathrm{CCH})$ and one Service Channel (SCH) interface with total channel duration of $100 \mathrm{~ms}$ with $50 \mathrm{~ms}$ per channel that switch periodically at $50 \mathrm{~ms}$ intervals.

To approximate real world radio propagation, we implemented a realistic radio propagation models in OPNET. The model is based on the Two-Ray Ground model with shadowing, where the parameters are set based on empirical testing of $802.11 \mathrm{p}$ radio modules [29]. The packet loss ratio is in the region of $40 \%$ for distances up to $100 \mathrm{~m}$ between the transmitter and receiver while the losses increase to $90 \%$ with distances of between $100 \mathrm{~m}$ and $150 \mathrm{~m}$, and $100 \%$ losses are achieved with distances beyond $200 \mathrm{~m}$.

For experimental investigation, we modelled an urban scenario using the road traffic simulator SUMO [30], where the scenario represents a topology of collector roads in a $5 \mathrm{~km}^{2}$ area in the Bishopstown district in Cork City, Ireland. The traffic model contained dynamically moving vehicles with varying speeds that are restricted to a maximum speed of $70 \mathrm{kmph}$ along 2-lane roads with a mixture of signalled intersections, traffic circles, and stop signs. The density of vehicles ranged from 10 to 140 vehicles per $\mathrm{km}^{2}$, which represented traffic flows at night time to peak time. Two types of emergency situations were investigated representing safety of life applications and low-priority hazard/environmental warning applications.

The first scenario emulates 3 accidents in 3 roads in low, medium, and high density road sections. Accidents can be detected by vehicles within $50 \mathrm{~m}$ of the accident location. A vehicle entering this $50 \mathrm{~m}$ sensing range detects and immediately invokes a broadcast relating to this emergency. A vehicle that is within this $50 \mathrm{~m}$ range when the accident occurs selects a random wait time over a uniformly distributed interval of $100 \mathrm{~ms}$ (corresponds to the WAVE SYNC INTERVAL) before broadcasting. This distributes the generation of broadcasts over the complete WAVE frame and randomises the intervals at which vehicles rebroadcast and 
lessen collisions due to broadcast storms. The broadcasting is repeated at $1 \mathrm{~s}$ intervals.

The second scenario was designed to focus on the throughput of the whole network and emulates an environmental network wide event, rain detection in this case. Each scheme was tested with different loads in the network. All vehicles detect the rain event uniformly distributed in time over $1 \mathrm{~s}$ and repeatedly broadcast every $1 \mathrm{~s}$.

\section{Performance Analysis}

In the simulated environment, only two types of messages are transmitted. Beacon messages WSA [1] were transmitted every $100 \mathrm{~ms}$ by each node, and safety messages were encapsulated in WSM [1] packets and broadcasted with the Minimum broadcast distance being set to $500 \mathrm{~m}$ and the Maximum hops being set to 10 hops. We collected the simulation results from 3 seeds with at least 200 runs for each seed. The metrics recorded from the experiments are outlined below and shown in Figures 5-10.

Network overview (Figure 5) - shown in this diagramis the mean number of 1st hop and 2nd hop neighbours that nodes have in the network. The diagram shows a limitation of ATPB and RR-ALOHA schemes.

Link Load (Figure 6) - this is calculated as the mean ratio of the number of nodes that transmit safety broadcast packet against the number of nodes that receive the packet. The lower this value the better as this indicates that fewer transmissions are needed to disseminate the broadcast packet.

End-to-End Delay (Figure 7) —-this is a measure of the mean time delay between the source of a safety message and the node that receives the broadcast last. This also covers the time delay created by time slots $\mathrm{CCH}$ TS and SCH TS in the Wave protocol. In the case of the SFR scheme, this is measured as the delay between the source node and the reception of last repetition broadcast.

Delivery Ratio (Figure 8)-this measure is dependent on the density of a network and it is the mean delivery ratio taken as the number of nodes inside an area that receive safety broadcast versus the number of nodes in that area. The area was defined by a source node as an area inside a circle with the source node at the centre, and the radius is defined by the Minimum broadcast distance. For the SFR scheme, this was measured based on the number of nodes inside the area that received safety broadcast (from any repetition) versus the number of nodes in the area.

Delivery Ratio versus Distance (Figure 9) - this shows the effect on the mean delivery ratio against increasing distance from the source up to the Minimum broadcast distance.

Throughput (Figure 10) - this measure was collected over the complete network and refers to global network events. All vehicles in a scenario detect a global event (e.g., raining) using sensors and all vehicles broadcast this event. The purpose of this measurement was to investigate the impact of the broadcast repetition interval, which was varied from 0.01 to 3 packets per second, on the delivery ratio (the number of nodes that receive the broadcast) in a network that was moderately busy, with 60 vehicles $/ \mathrm{km}^{2}$.

\section{Theoretical and Experimental Results}

6.1. Theoretical Results. We compare the proposed PACK scheme with 3 existing schemes, namely SFR, ATPB, and RR-ALOHA. All the schemes were overlaid on the SRMB broadcasting protocol. According to the WAVE standard [1], time was divided to frames (Sync interval) with a length of $100 \mathrm{~ms}$. Each frame contains two slots the Control Channel (CCH TS) and the Service Channel (SCH TS) time slots, each with a length of $50 \mathrm{~ms}$. Each of these slots begins with a Guardian time of $5 \mathrm{~ms}$ to allow a unit to switch from one channel to another. In the Guardian time interval, no messages can be sent. Beacon messages and safety messages were sent only in CCH TS after the Guardian time. If a safety message was sent in CCH TS, the beacon message was omitted to prevent overloading the medium.

For repeated broadcasting of an event (local, global), the invoking of safety messages was uniformly distributed across the Sync interval with a length of $100 \mathrm{~ms}$. If a safety message was invoked during the SCH TS $50 \mathrm{~ms}$ interval or Guardian time $5 \mathrm{~ms}$ duration, then it waited until the beginning of the $\mathrm{CCH}$ TS where it was immediately transmitted. A mean time delay $T_{H \_M A C}$ for waiting emergency data (WSM) at the upper MAC layer before being passed to the lower MAC layer to access the CCH TS is calculated as per the following equation:

$$
\overline{T_{H_{-} \mathrm{MAC}}^{\mathrm{SRMB}}}=\frac{T_{\mathrm{SCH}+\mathrm{G}}}{T_{\text {sync }}} \cdot \frac{T_{\mathrm{SCH}+\mathrm{G}}}{2} \approx 15 \mathrm{~ms} .
$$

(i) $T_{\mathrm{SCH}+G}$ is the time in length of SCH TS (50 ms) plus Guardian time (5 ms) when emergency data cannot be sent.

(ii) $T_{\text {sync }}$ is the length of Sync interval $100 \mathrm{~ms}$ specifies in Wave [1].

The Mean theoretical overall time delay for multihop broadcasting $T_{\mathrm{SRMB}}$ is calculated as per equation (5), which is derived from (1), (2), and (4) as follows:

$$
\begin{aligned}
& T_{\mathrm{SRMB}}=T_{H_{-} \mathrm{MAC}}^{\mathrm{SRMB}}+H \cdot\left(T_{\text {slot }}(J)+T_{L_{-} \mathrm{MAC}}\right) \\
& \overline{T_{\mathrm{SRMB}}} \approx 18 \mathrm{~ms} .
\end{aligned}
$$

(It presumes that all transmissions were made in one $\mathrm{CCH}$ TS. Otherwise the $T_{H \_M A C}$ was extended to $55 \mathrm{~ms}$ (length of SCH TS and Guardian time).)

(i) $L_{\text {DATA }}$ in (1) is the size in bits of an emergency packet (WSM) with a value of 368 bits.

(ii) $H$ is the mean number of hops and is set to 6 . The number was taken from mean number of hops in the simulations that increased with increasing density.

(iii) It is presumed that $T_{\text {slot }}$ with $J \in(1 \leq N)$ is the delay applied mainly at the origin of the broadcast, 
where broadcasts are sent in different sectors based on the priority of the MPR nodes. Here, $J$ represents the average number of MPR nodes per hop, based on simulation evaluation this was set to $J=1.5$.

6.1.1. Pack. In case of using the PACK scheme, the overall multihop delay $T_{\text {SPACK }}$ is slightly increased due to the following repetitions:

$$
\begin{aligned}
& T_{\mathrm{PACK}}=T_{\mathrm{SRMB}}+k \cdot T_{\mathrm{rep}}, \\
& \overline{T_{\mathrm{PACK}}} \approx 22 \mathrm{~ms},
\end{aligned}
$$

where $k$ is the number of repetitions. This value depends on the data traffic on the physical medium, where in less busy network the repetition value was approximately one repetition per the complete broadcast and this went up to approximately 7 for busy networks. For theoretical estimation, we set $k=2.5$, which is compared to medium busy network.

The SRMB+PACK scheme increased end-to-end delay of SRMB protocol by $22 \%$ ( $18 \mathrm{~ms}$ compared to $22 \mathrm{~ms}$ ).

6.1.2. Synchronous Fixed Retransmission (SFR). In case of using the SFR scheme, the overall multihop delay $T_{\text {SFR }}$ was calculated as follows:

$$
\begin{aligned}
& T_{\mathrm{SFR}}=T_{\mathrm{SRMB}}+k \cdot T_{\text {SFR_slot }}, \\
& \overline{T_{\mathrm{SFR}}} \approx 23 \mathrm{~ms} .
\end{aligned}
$$

(i) $k$ is the mean number of broadcast repetitions equally distributed from 0 to 9 as specified by the SFR scheme.

(ii) $T_{\text {SFR_slot }}$ is a slot in length of $1 \mathrm{~ms}$ specifies by SFR scheme.

The SFR scheme increased end-to-end delay of SRMB protocol by $28 \%$ and by $5 \%$ when compared against SRMB+PACK.

6.1.3. Adaptive Transmission Power of Beacons (ATPBs). The theoretical overall time delay of multihop broadcasting was kept the same as in SRMB protocol. From the perspective of broadcasting delay, the ATPB and SRMB schemes work on the same principle. ATPB only affects the transmission power of the beacons and does not straight impact on the dissemination of emergency (WSM) data.

6.1.4. Reliable Reservation-ALOHA (RR-ALOHA). In RRALOHA, the beacon (WSA) contained a list of all time slots, where each entry relates to particular time slot. Each entry in the list had a size of 11 bits and contained information relating to the state of the channel (busy or idle) and the short MAC address of the node transmitting on that time slot. Because we implemented RR-ALOHA over the WAVE standard, we had to derive the maximum number of slots first. The size of beacons $L_{\text {DATA }}$ used by RR-ALOHA was calculated in as follows:

$$
\begin{aligned}
& L_{\mathrm{DATA}}^{\mathrm{RR}-\mathrm{ALOHA}}=L_{\mathrm{MAC}}+L_{\mathrm{WSA}}+L_{\mathrm{RR}-\mathrm{ALOHA}}, \\
& L_{\mathrm{RR}-\mathrm{ALOHA}}=11 \text { bits } \cdot S,
\end{aligned}
$$

where $L_{\text {MAC }}$ is the size of the MAC header with 272 bits, and $L_{\text {WSA }}$ is the size of the WSA beacons with length of 480 bits. From a knowledge of the maximum available time of $45 \mathrm{~ms}$ in the CCH TS and from maximum transmission delay (1), we determine that the maximum number of time slots $S$ used by RR-ALOHA is 90 with length of a slot being $0.5 \mathrm{~ms}$. The overhead $L_{\text {RR-ALOHA }}$ was calculated as 11 bits $\times 90$ time slots, which is 990 bits.

The mean theoretical overall multihop delay $T_{\text {RR-ALOHA }}$ was calculated as follows:

$$
\begin{aligned}
& \overline{T_{H \_ \text {MAC }}^{\mathrm{RR}}} \approx 50 \mathrm{~ms}, \\
& T_{\mathrm{RR}-\mathrm{ALOHA}}=T_{H_{-} \text {MAC }}^{\mathrm{RR}-\mathrm{ALOHA}} \cdot H+T_{L_{-} \mathrm{MAC}}, \\
& \overline{T_{\mathrm{RR}-\mathrm{ALOHA}}} \approx 300 \mathrm{~ms} .
\end{aligned}
$$

The delay $T_{\text {RR-ALOHA }}$ depends on the number of hops $H$ and how many retransmit nodes are chosen. Theoretically, with 10 hops ( 10 hops in the maximum number of allowable hops for a broadcast) the delay can vary from $18 \mathrm{~ms}$ (see (5)) to $1000 \mathrm{~ms}$ (see (9)) depending on the selecton of forwarding hops and their time slot.

RR-ALOHA gives the longest delay, 17 times higher than SRMB and 14 times higher than SRMB+PACK and SFR.

6.2. Experimental Results. All the results presented are represented by mean values for individual data points which are averaged over approximately 600 values with 3 seeds. The data sets in most cases have a skewed distribution, so it is preferable to use the first and third quartiles $\left(q_{25}, q_{75}\right)$ as descriptive statistics.

6.2.1. Network Overview. Network overview (Figure 5) -this shows the mean number of 1-hop and 2-hop neighbours that nodes have in the network. In [21], for the ATP scheme the neighbour threshold is set to 30 nodes, meaning that if a node has more than 30 neighbours, then the node should change its transmission power which would then affect the broadcasting performance. As can be seen in Figure 5, the number of neighbours exceeds 30 between $x=40$ and $x=60$ (vehicles $/ \mathrm{km}^{2}$ ). The RR-ALOHA protocol uses time slots, where the number of time slots, was set to 90 using (1) and (8). The number of 1 st hop and 2 nd hop neighbours exceeds the maximum number of slots, that is, 90 at $x=60$ (vehicles $/ \mathrm{km}^{2}$ ), beyond this density some nodes will have to share the same time slot.

The results show a limitation of the ATPB and RRALOHA as the number of neighbouring nodes can affect the broadcast performance. SRMB+PACK and SFR are not restricted by number of neighbours and can work across all neighbour densities. 


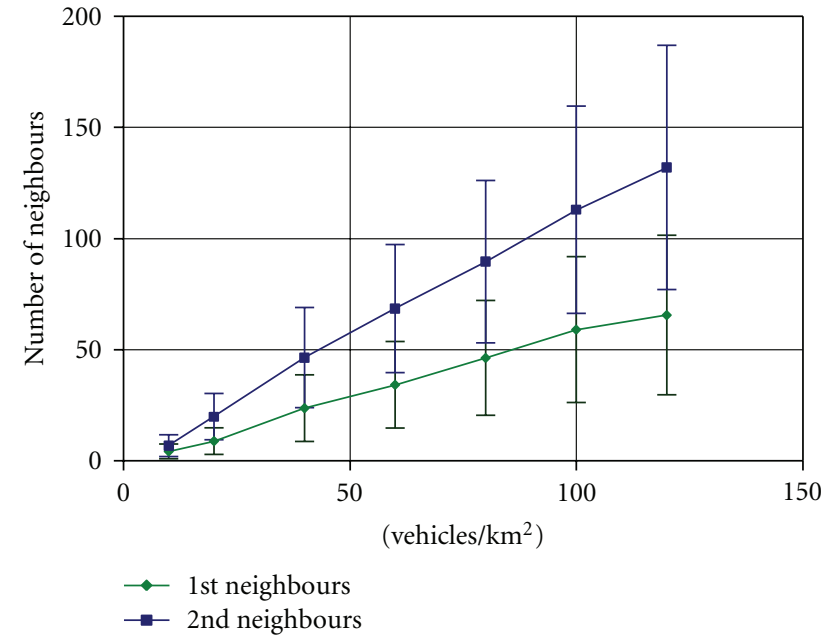

FIGURE 5: Network overview shows the mean number of 1st hop and 2 nd hop neighbours that nodes have in the network.

6.2.2. Link Load. Link Load (Figure 6) showed that all schemes (except SFR because of repetitions) have a rapidly decreasing link load trend. As the vehicle density increases, the network connectivity goes from sparsely connected to well connected. After SFR, the SRMB protocol performs the next worst in terms of link load (with a Link Load Ratio mean value of \#LL $=0.25$, with 1 st and $3 \mathrm{rd}$ quartiles being $q_{25}=0.14, q_{75}=0.25$, taken at the highest density of vehicles with $x=140$ vehicles $/ \mathrm{km}^{2}$ ) in denser networks. The PACK scheme in lower density networks performs marginally poorer $(5 \%$, this drop in performance is attributed to the repetition of broadcasts for unacknowledged sectors) than SRMB with \#LL $=0.77$, $q_{25}=0.5$, and $q_{75}=1$, at a vehicle density $x=10 / \mathrm{km}^{2}$ in less busy networks. For higher density networks with $x=140$ vehicles $/ \mathrm{km}^{2}$, values of \#LL $=0.22, q_{25}=$ 0.18 , and $q_{75}=0.27$ are achieved, and this represents an improvement of $12 \%$ when compared with SRMB. For more saturated networks, the pseudoacknowledgements used by PACK to acknowledge sectors reduce the probability of nonMPR nodes rebroadcasting and thus reduce the probability of collisions which results in fewer transmissions in the congested medium. The ATPB and SRMB schemes have a similar performance as the power control aspect of ATPB only applies to the beacons. The best performance across all densities was achieved by RR-ALOHA as expected with a $40 \%$ improvement over SRMB at a vehicle density of $x=$ $140 / \mathrm{km}^{2}$ and $\# \mathrm{LL}=0.15, q_{25}=0.12$, and $q_{75}=0.15$. This performance is attributed to the fact that RR-ALOHA uses one slot per node transmissions and will always outperform CSMA/CA methods, on which the other schemes are based. The better performance in terms of link load is offset by the poor end-2-end delay and throughput achieved with RRALOHA. The worst performance, that is, the greatest number of transmissions was attributed to SFR, which significantly differs from the other schemes. In the lightest density, SFR reached a value ( $\# \mathrm{LL}=4, q_{25}=2, q_{75}=6$, and density $x=10) 5$ times greater than SRMB. In the heaviest density

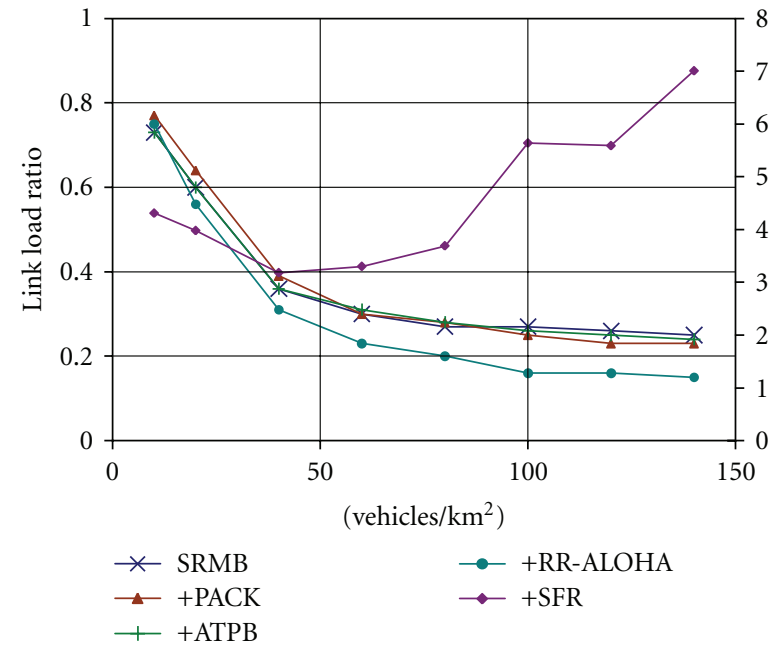

FIgURE 6: Link Load Ratio is calculated as the mean ratio of the number of nodes that transmit a broadcast packet against the number of nodes that receive the packet. Second right $y$ axis is for SFR scheme, which significanty differ from the others.

network, SFR flooded the network, which led to rapidly increasing unsuccessful transmissions ( $\# \mathrm{LL}=7, q_{25}=0.5$, $q_{75}=2$, and density $x=140$ ) with values 30 times greater than in SRMB.

The link load results show that all schemes (except SFR) perform broadcasting with a very low number of transmissions and decrease the number with increasing density of vehicles as network increasing in connectivity due to a larger number of nodes. At higher densities, SFR flooded network because of repetitions and is actually worse than using a simple flooding protocol making SFR unsuitable for VANETs.

6.2.3. End-to-End Delay. End-to-End Delay (Figure 7)-As expected due to their similar operation, the results for endto-end delay showed that the SRMB protocol and the ATPB scheme maintain the same relatively constant short time delay (End-to-End Delay, $\# \mathrm{EE}=20 \mathrm{~ms}, q_{25}=4, q_{75}=37$, and density $x=140$ ), which matched the theoretical result achieved with (5). In comparison, the PACK method had a slightly increased delay across all densities from lighter densities (\#EE $=18 \mathrm{~ms}, q_{25}=0.4, q_{75}=33$, and density $x=10$ ) with a deterioration in performance when compared with SRMB of $12 \%$ and in larger densities $(\# \mathrm{EE}=33 \mathrm{~ms}$, $q_{25}=8, q_{75}=50$, and density $\left.x=140\right)$ a deterioration of $50 \%$ again comparing to SRMB. Using (6) and a repetition factor of 2.5 and looking at a medium density network with $x=40 / \mathrm{km}^{2}$, theoretical results matched experimental result $\left(\# \mathrm{EE}=22 \mathrm{~ms}, q_{25}=4, q_{75}=39\right.$, and density $\left.x=40\right)$. The SFR scheme gave the 2nd longest delay across a lowdensity network (\#EE $=22 \mathrm{~ms}, q_{25}=5, q_{75}=40$, and density $x=10$ ) to a high-density network (\#EE $=47 \mathrm{~ms}$, $q_{25}=11, q_{75}=60$, and density $x=140$ ) with a deterioration in performance ranging from $40 \%$ to $240 \%$ when compared against SRMB. Using (7), the theoretical end-to-end delay 


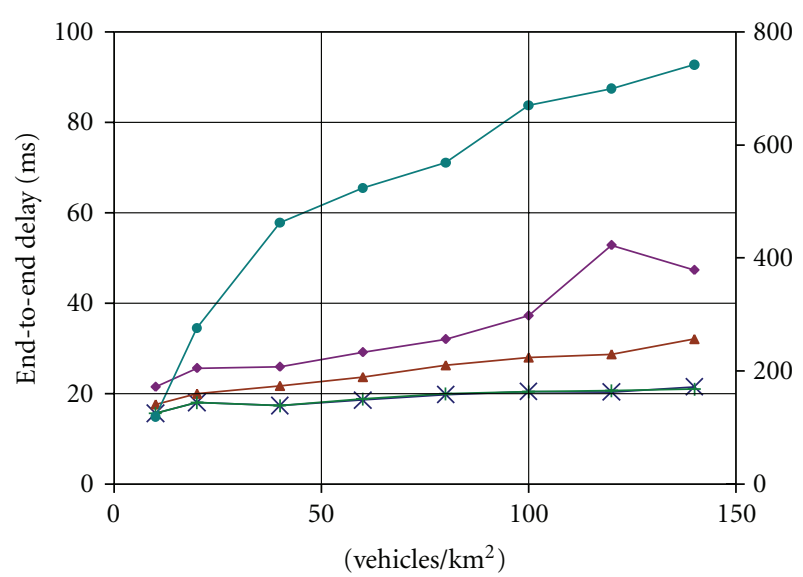

Figure 7: End-to-End Delay is a measure of the mean time delay between the source of a safety message and the node that receives the broadcast last. Second right $y$ axis is for RR-ALOHA scheme, which significanty differ from the others. Label is the same as at Figure 6.

does not match the empirical result. Equation (7) is derived using the maximum transmission time $T_{L_{-} M A C}$ from (1) which does not consider a saturated case (i.e., collisions are not considered). Equation (1) is valid only for lightly loaded networks. In more dense networks if a transmission on the medium is detected while a node is in Backoff, a new Backoff time is set and the transmission delay (1) is increased. With the SFR protocol, we have an increasing load on the physical medium as a consequence of repetitions that saturate the network and lead to collisions. The longest delay is given by RR-ALOHA across all densities from the lowest (\#EE = $120 \mathrm{~ms}, q_{25}=40, q_{75}=176$, and density $\left.x=10\right)$ to highest (\#EE $=740 \mathrm{~ms}, q_{25}=580, q_{75}=920$, and density $x=140$ ) with a deterioration from 7.5 times to 35 times that of SRMB. Using (9), we see that the theoretical result depended strongly on the number of hops and the selection of the next hops based on their time slots. This variation was described in Section 6.1.4 and matched experimental results.

The results showed that the SRMB, ATPB, PACK, and SFR schemes reach a fraction of driver reaction time (around $0.05 \mathrm{~s}$ of $0.7 \mathrm{~s}[31])$. On the basis of the results, we show that these schemes in terms of end-to-end delay are appropriate for VANETs. As RR-ALOHA has prohibitively long endto-end delays across all densities, we conclude that this method based on comparison with driver reaction speeds is unsuitable for emergency data dissemination in VANETs.

6.2.4. Delivery Ratio. Delivery Ratio (Figure 7)—Results showed that the SRMB protocol reached relatively constant values for Delivery Ratio, $\# \mathrm{DR}=0.62, q_{25}=0.48, q_{75}=0.90$, and density $x=40$ to \#DR $=0.61, q_{25}=0.43, q_{75}=$ 0.92 , and density $x=140$. Similar results were achieved with ATPB and acknowledged that sparing communication capacity by decreasing transmit power of beacons did not have significant effect on delivery ratio. The PACK method in low-density network gave values of ( $\# \mathrm{DR}=0.35, q_{25}=$ $0.18, q_{75}=0.45$, density $x=10$ ) and in high density

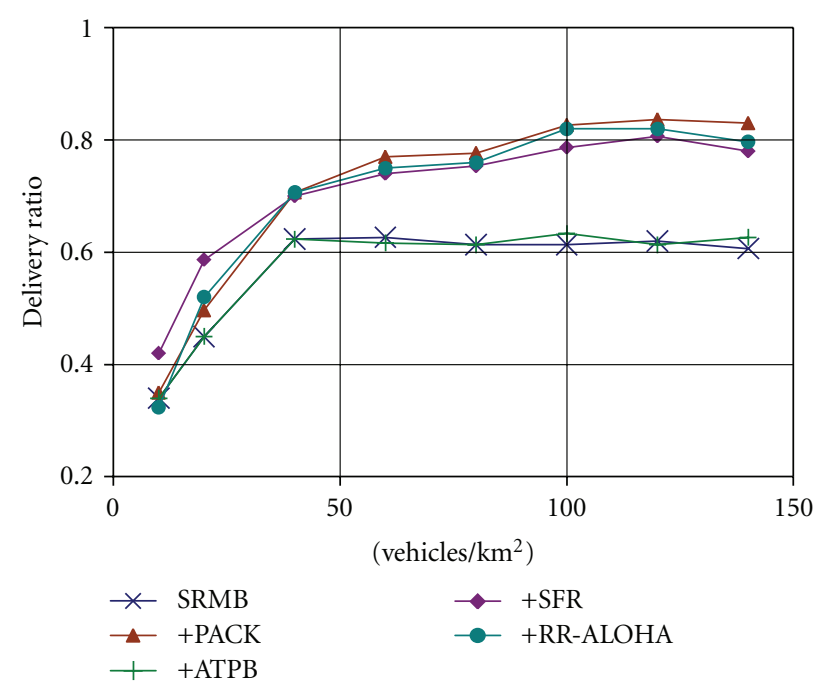

FIGURE 8: Delivery Ratio is a measure of the mean delivery ratio taken as the number of nodes inside an area that receive a broadcast versus the number of nodes in that area.

gave $\left(\# \mathrm{DR}=0.83, q_{25}=0.77, q_{75}=0.96\right.$, and density $x=140)$. These results reflect improvements of $2 \%$ to $36 \%$ when comparing against SRMB from medium- to highdensity networks $\left(\# \mathrm{DR}=0.70, q_{25}=0.57, q_{75}=0.97\right.$, and medium density $x=40$ ) with PACK improving overall other methods in medium- to high-density networks. SFR gives the best performance in lower density networks because of the repetitions in a sparsely connected network (\#DR = $0.42, q_{25}=0.30, q_{75}=0.55$, and density $x=10$ ) with improvements of $32 \%$ over SRMB. In medium busy densities $\left(\# \mathrm{DR}=0.70, q_{25}=0.60, q_{75}=0.98\right.$, and density $\left.x=40\right)$, SFR has a slight deterioration of $4 \%$ when compared to PACK and in the highest density ( $\# \mathrm{DR}=0.78, q_{25}=0.77$, $q_{75}=0.97$, and density $x=140$ ), the decline in performance falls to $6 \%$ when compared to PACK. The RR-ALOHA scheme gave a slightly poorer results when compared to PACK (low density $\left(\# \mathrm{DR}=0.33, q_{25}=0.18, q_{75}=0.45\right.$, and density $x=10$ ) to high density (\#DR $=0.80, q_{25}=0.76$, $q_{75}=0.96$, and density $x=140$ ) with a $5 \%$ decline in performance.

The results show that PACK, SFR, and RR-ALOHA significantly improved delivery ratio across all densities with ATPB giving a performance again similar to SRMB. Again this shows the unsuitability of the ATPB protocols for reliable broadcasting in VANETs as it only refers to beacon frames.

6.2.5. Delivery Ratio versus Distance. Delivery Ratio versus Distance (Figure 8) - these results were captured at a density of 60 vehicles $/ \mathrm{km}^{2}$ (medium busy network) and showed that for all schemes the Delivery Ratio fell of with increasing distance. Again SRMB and ATPB give similar results. PACK, SFR, and RR-ALOHA improve on SRMB and give very similar values up to a distance, $x=250 \mathrm{~m}$ from a sender, with an improvement of $18 \%$ over SRMB. 


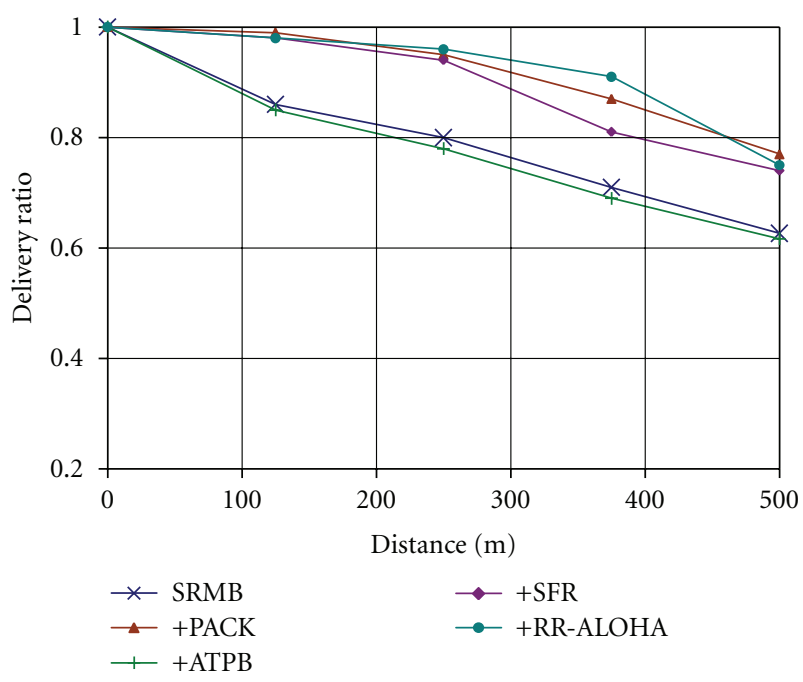

Figure 9: Delivery Ratio shows the effect on the mean Delivery Ratio against increasing distance.

Further, from the sender at a distance $x=350 \mathrm{~m}$, SFR gives an improvement of $14 \%$, PACK gives $22 \%$ and RRALOHA 28\% over SRMB. On the boundary of the Minimum broadcast distance $(500 \mathrm{~m})$, improvements with SFR were $23 \%$, RR-ALOHA 25\%, and PACK 28\% over SRMB.

The results showed that with increasing distance the delivery ratio falls off, but again SFR, PACK, and RR-ALOHA improve broadcasting performance.

6.2.6. Throughput. Throughput (Figure 10)—results showed that all schemes have a decreased delivery ratio for broadcasts with increasing load in the network. As before, SRMB and ATPB perform similarly. SRMB (likewise ATPB) give the lowest throughput ratio \#TP $=0.45, q_{25}=0.27, q_{75}=0.72$, and load $x=0.01$ packets $/ 1 \mathrm{~s}$ under low load and this decreases \#TP $=0.32, q_{25}=0.04$, and $q_{75}=0.53$, with increasing load $x=3$ packets $/ 1 \mathrm{~s}$ (high load). In low load, $x=0.01$ packets $/ 1 \mathrm{~s}$ RR-ALOHA has \#TP $=0.49$, which improves on SRMB by $9 \%$ and maintained a trend similar to PACK until reaching a moderately loaded network $x=0.3$ packets $/ 1 \mathrm{~s}$, where \#TR $=0.25, q_{25}=0.02, q_{75}=0.43$, and load $x=3$ packets/ $1 \mathrm{~s}$ fell to 22\% below SRMB. This performance deterioration is attributed to the fact that RRALOHA uses TDMA access and will always perform worse than CSMA/CA access, on which the other schemes are based. SFR reached the highest values with $\# T R=0.58, q_{25}=$ 0.30 , and $q_{75}=0.87$ under low load $x=0.01$ packets $/ 1 \mathrm{~s}$ and deteriorated $\# T R=0.42, q_{25}=0.30$, and $q_{75}=0.73$ under greater load $x=3$ packets $/ 1 \mathrm{~s}$ due to saturating the network with repeat broadcasts but still improved on SRMB by $31 \%$. The PACK scheme reached values similar to RR-ALOHA under low load. With increasing load, the PACK improves on these with $\# T R=0.45, q_{25}=0.22$, and $q_{75}=0.68$, high load $x=3$ packets $/ 1 \mathrm{~s}$ giving an improvement of $40 \%$ over SRMB, $80 \%$ over RR-ALOHA, and 7\% over SFR.

The results showed that with increasing load the throughput ratio dropped across all schemes. TDMA-based

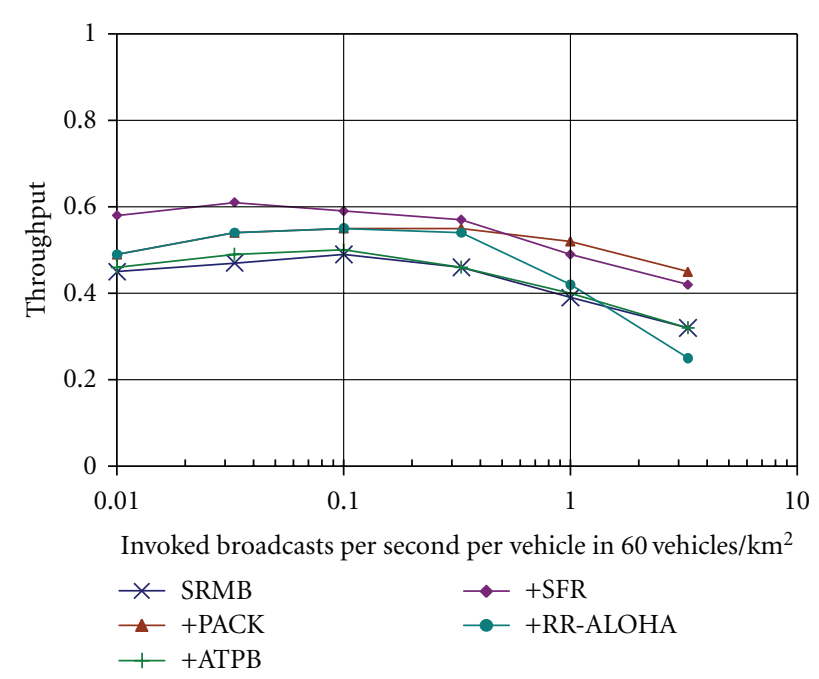

Figure 10: Throughput measure an impact of the broadcast repetition interval on the delivery ratio.

channel access in RR-ALOHA shows reduced throughput against CSMA/CA-based access in highly loaded networks. SFR scheme showed decreased throughput due to overloading of network with many repetitions but still maintains a higher performance then SRMB. The PACK scheme in moderate to highly loaded networks gives a better performance than the SFR, RR-ALOHA, and SRMB (ATPB). These advantages of the PACK algorithm can be further highlighted when safety application is required to report with a higher rate $(<1 \mathrm{~s})$ as it can maintain high delivery ratio for moderateto high-density networks (e.g., vehicle platooning).

\section{Discussion, Conclusions, and Future Work}

In this paper, we have concentrated on techniques that increase reliability of a multihop broadcast protocol. We have proposed the Pseudoacknowledgments (PACK) scheme that improves reliability in multihop broadcasting protocols by repeating broadcast transmissions on unsuccessful links. The scheme was compared with existing mechanisms over an urban scenario using the network simulator tool OPNET [10] with an empirical-based propagation model [29], realistic mobility patterns using the road traffic simulator SUMO [30], and the Wave [1] standard. All schemes were overlaid on the low-latency p-persistent CSMA/CA broadcasting protocol called the Slotted Restricted Mobility-Based (SRMB) protocol.

The scenarios were designed to test safety-related data dissemination and measured relevant broadcasting statistics: link load, end-to-end delay, delivery ratio, and throughput. From our results, we draw the following conclusions and identify tasks for future work.

(i) Changing transmission power of beacons does not have a significant effect on performance of broadcasting, as has been demonstrated by examining the performance of ATPB. 
(ii) Repeating broadcasts leads to increased delivery ratio, but it also increases the number of transmissions in the network. This can lead to flooding the network with repetitions and can decrease the delivery ratio in denser networks. The throughput results show that SFR scheme can easily saturate the network under higher loads which leads to a rapidly decreasing delivery ratio. The redundancy incurred as a consequence of repetitions which can lead to flooding makes this scheme unsuitable for VANETs.

(iii) Using small time slots for broadcasts leads to a high delivery ratio due to minimum collisions in particular slots but this increases end-to-end delay as broadcasts are delayed at successive rebroadcast nodes. In the case of RR-ALOHA, the delay reaches large values that cannot be tolerated for safety-related data dissemination. Another disadvantage of slotting is that it decreases throughput in densely loaded network which corresponds with the throughput performance when comparing CSMA/CA access with ALOHA access. When the number of nodes exceeds the maximum number of available slots, nodes must share slots. However, this limitation does not have a significant effect on the broadcasting performance in our simulations.

(iv) In Summary, PACK in moderate-to high-density networks achieves an increase in delivery ratio of approximately $5 \%$ over SFR and 3\% over RR-ALOHA while this may appear to be a minor increment RR-ALOHA is unsuitable for VANETs due to its excessive end-to-end delay, for example, $741 \mathrm{~ms}$ for RRALOHA against $32 \mathrm{~ms}$ for PACK in high-density networks. While SFR has a tolerable end-to-end delay for VANETs, it does so at the expense of saturating the network with repeat broadcasts and this is highlighted by the link load metric which at a vehicle density of $140 / \mathrm{km}^{2}$ is 35 times higher than of PACK. While PACK and SFR give similar delivery ratio metrics, SFR does so at the expense of excessive bandwidth usage in comparison to PACK. Furthermore, due to less saturating of the network PACK achieved the highest throughput in moderate to high density networks. This makes PACK suitable for applications that need to frequently report (e.g., vehicle platooning, crashed vehicle detection).

(v) From the experimental results presented in this paper, we can conclude that the PACK mechanism increases the reliability of multihop broadcasting and is suitable for safety-related data dissemination. In terms of end-to-end delay and bandwidth savings, PACK outperforms RR-ALOHA and SFR, respectively, making PACK a more reliable protocol for safety data dissemination in VANETs.

Although SRMB+PACK protocol has been primarily tested on a specific case of safety application (accident reporting), the SRMB+PACK mechanism can be used as a multihop data dissemination mechanism for a range of applications that require high packet delivery and low latency in very dynamic ad hoc networks. The SRMB+PACK protocol could be used in route discovery for reactive routing protocols in VANETs. From the route discovery, perspective routes would be built based on latency, bandwidth consumption, and mobility of nodes in the source-destination path. Nodes with similar mobility behaviour (speed, motion vector) would be selected as intermediate hops as this supports the generation of stable routes and reduces route maintenance overhead.

\section{References}

[1] "Intelligent Transportation Systems Standards Fact Sheet," IEEE 1609-Family of Standards for Wireless Access in Vehicular Environments (WAVE), ITS Standards Program.

[2] http://europa.eu/legislation_summaries/transport.

[3] http://ec.europa.eu/information_society/eeurope/2005/index_ en.htm.

[4] W. Chen, R. K. Guha, T. J. Kwon, J. Lee, and I. Y. Hsu, "A survey and challenges in routing and data dissemination in vehicular ad-hoc networks," in Proceedings of the IEEE International Conference on Vehicular Electronics and Safety, pp. 328-333, Columbus, Ohio, USA, September 2008.

[5] M. Torrent-Moreno, D. Jiang, and H. Hartenstein, "Broadcast reception rates and effects of priority access in 802.11based vehicular ad-hoc networks," in Proceedings of the 1st ACM International Workshop on Vehicular Ad Hoc Networks (VANET '04), pp. 10-18, Philadelphia, Pa, USA, 2004.

[6] G. Korkmaz, E. Ekici, F. Ozguner, and U. Ozguner, "Urban multiHop broadcast protocol," in Proceedings of the 1st ACM International Workshop on Vehicular Ad Hoc Networks, pp. 7685, Philadelphia, Pa, USA, 2004.

[7] M. Sun, W. Feng, T. Lai, K. Yamada, H. Okada, and K. Fujimura, "GPS-based message broadcasting for inter-vehicle communication ," in Proceedings of the International Conference on Parallel Processing, pp. 279-286, Toronto, Canada, 2000.

[8] M. Heissenbuttel, T. Braun, M. Walchli, and T. Bernoulli, "Optimized stateless broadcasting in wireless multi-hop networks," in Proceedings of the 25th IEEE International Conference on Computer Communications (INFOCOM '06), pp. 1-12, Barcelona, Spain, April 2006.

[9] W. Si and C. Li, "RMAC: a reliable multicast MAC protocol for wireless ad hoc networks," in Proceedings of the International Conference on Parallel Processing (ICPP '04), pp. 494-501, August 2004.

[10] http://www.opnet.com.

[11] J. Peng, "Slim ARQ for reliable broadcasting in wireless LANs," in Proceedings of the IEEE Wireless Communications and Networking Conference (WCNC'08), pp. 2164-2169, 2008.

[12] S.-T. Sheu, Y. Tsai, and J. Chen, "A highly reliable broadcast scheme for IEEE 802.11 multi-hop ad hoc networks," in Proceedings of the IEEE International Conference on Communications (ICC '02), vol. 1, pp. 610-615, New York, NY, USA, 2002.

[13] K. Tang and M. Gerla, "Random access MAC for efficient broadcast support in ad hoc networks," in Proceedings of the IEEE Wireless Communications and Networking Conference (WCNC '00), pp. 454-459, Los Angeles, Calif, USA, 2000.

[14] http://www.ietf.org/rfc/rfc3626.txt. 
[15] F. Hassanzadeh, Reliable broadcast of safety messages in vehicular ad hoc networks, M.S. thesis, Graduate Department of Electrical and Computer Engineering University of Toronto, Toronto, Canada, 2008.

[16] F. Borgonovo, A. Capone, M. Cesana, and L. Fratta, "RRALOHA, a Reliable R-ALOHA broadcast channel for ad-hoc inter-vehicle communication networks," 2002, ftp://ftp.elet .polimi.it/users/Flaminio.Borgonovo/PUBBC/2002medhoc .pdf.

[17] http://www.cartalk2000.net.

[18] Q. Xu, T. Mak, J. Ko, and R. Sengupta, "Medium access control protocol design for vehicle-vehicle safety messages," IEEE Transactions on Vehicular Technology, vol. 56, no. 2, pp. 499518, 2007.

[19] Q. Xu, R. Senguptay, T. Mak, and J. Ko, "Vehicle-to-vehicle safety messaging in DSRC," in Proceedings of the 1st ACM International Workshop on Vehicular Ad Hoc Networks, pp. 1928, 2004.

[20] F. Farnoud and S. Valaee, "Repetition-based broadcast in vehicular ad hoc networks in Rician channel with capture," in Proceedings of the 27th Conference on Computer Communications (INFOCOM '08), pp. 1-6, 2008.

[21] L. Yang, J. Guo, and Y. Wu, "Channel adaptive one hop broadcasting for VANETs," in Proceedings of the 11th IEEE Conference on Intelligent Transportation Systems (ITSC '08), pp. 369-374, Beijing, China, 2008.

[22] M. Torrent-Moreno, P. Santi, and H. Hartenstein, "Distributed fair transmit power adjustment for vehicular ad hoc networks," in Proceedings of the 3rd Annual IEEE Communications Society on Sensor and Ad Hoc Communications and Networks (Secon '06), vol. 2, pp. 479-488, Reston, Va, USA, 2006.

[23] M. Koubek, S. Rea, and D. Pesch, "Effective emergency messaging in WAVE based VANETs," in Proceedings of the 1st International Conference on Wireless Access in Vehicular Environments (WAVE'08), December 2008.

[24] http://www.ietf.org/rfc/rfc3561.txt.

[25] O. Tonguz, N. Wisitpongphan, F. Bai, P. Mudalige, and V. Sadekar, "Broadcasting in VANET," in Proceedings of the Mobile Networking for Vehicular Environments (MOVE '07), pp. 7-12, May 2007.

[26] “802.11e-2005, IEEE Standard for Information technologytelecommunications and information exchange between systems-local and metropolitan area networks-specific requirements-part 11: Wireless LAN Medium Access Control (MAC) and Physical Layer (PHY) specifications Amendment 8: Medium Access Control (MAC) Quality of Service Enhancements," 2005.

[27] J. Mittag, F. Thomas, J. Härri, and H. Hartenstein, "A comparison of single- and multi-hop beaconing in VANETs," in Proceedings of the 6th ACM International Workshop on Vehicular Inter-Networking (VANET'09), pp. 69-78, 2009.

[28] W. Crowther, R. Rettberg, D. Waldem, S. Ornstein, and F. Heart, "A system for broadcast communications: reservation ALOHA," in Proceedings of the 6th Hawaii International Conference on System Sciences, January 1973.

[29] O. Brickley, M. Koubek, S. Rea, and D. Pesch, "A network centric simulation environment for CALM-based cooperative vehicular systems," in Proceedings of the 3rd International Conference on Simulation Tools and Techniques (SIMUTools '10), 2010.

[30] http://sumo.sourceforge.net.

[31] M. Green, "“How long does it take to stop?” Methodological analysis of driver perception-brake times," Transportation Human Factors, vol. 2, no. 3, pp. 195-216, 2000. 